\title{
FAST AND ACCURATE: HIGH-SPEED METROLOGICAL LARGE RANGE AFM FOR SURFACE AND NANOMETROLOGY
}

\author{
Gaoliang Dai ${ }^{1}$, Pei Liu ${ }^{1}$, Ludger Koenders ${ }^{1}$, Jens Fluegge ${ }^{1}$, Matthias Hemmleb ${ }^{2}$ \\ ${ }^{1}$ Physikalisch-Technische Bundesanstalt (PTB), 38116 Braunschweig, Germany \\ ${ }^{2} \mathrm{~m} 2 \mathrm{c}$, Potsdam, Germany
}

\begin{abstract}
Low measurement speed remains as a major shortcoming of the scanning probe microscopic techniques. It leads not only to a low measurement throughput, but also to a significant measurement drift over the long measurement time needed (up to hours or even days). In this paper, development of a high speed metrological large range atomic force microscope (HS Met. LR-AFM) with a capable measurement speed up to $1 \mathrm{~mm} / \mathrm{s}$ is presented. $\mathrm{In}$ its design, a high accurate nanopositioning and nanomeasuring machine (NMM) is combined with a high dynamic flexure hinge piezo stage to move sample. The AFM output signal is combined with the position readouts of the piezo stage and the NMM to derive the surface topography. This design has a remarkable advantage that it well combines different bandwidth and amplitude of different stages/sensors, which is required for high speed measurements. While the HS Met. LR-AFM significantly reduces the measurement time while maintains (or even improves) the metrological performance than the previous Met. LR-AFM, its application capabilities are extended significantly. Two application examples, the realisation of reference areal surface metrology and the calibration of a kind 3D nano standards, have been demonstrated in the paper in detail.
\end{abstract}

\section{INTRODUCTION}

Since the invention of the scanning tunneling microscope (STM) in 1982 [1] and of the scanning force microscope (SFM) in 1986 [2] a large family of scanning probe microscopic (SPM) techniques has been developed and widely applied today for various measurement and analytic applications of nanotechnologies. Compared to optical microscopic techniques, the SPM has much high lateral resolution typically defined by the tip radius (down to $1 \mathrm{~nm}$ or even below). Compared to the scanning charged particle (electron and ion) microscopes, SPM is capable of measuring not only in vacuum, but also in liquid and air environment. In addition, the SPM technique is almost non-destructive due to small tip-sample interaction force and offers high resolution height information of the measured structures.

However, as a scanning probe method the low measurement speed remains as a major shortcoming of the SPM technique today. Taking a typical Atomic Force Micrscope (AFM) with a tip scanning speed of $30 \mu \mathrm{m} / \mathrm{s}$ as an example, it will take approximately 1 hour to finish 
an image with a size of $100 \mu \mathrm{m}$ x $100 \mu \mathrm{m}$ by 1024 x 1024 pixels. This situation becomes more challenging for a metrological large range microscope which has a capable measurement volume of $25 \mathrm{~mm} \times 25 \mathrm{~mm} \times 5 \mathrm{~mm}$ (x, y, z) [3]. As the low speed leads not only to a low measurement throughput, but also to a significant drift over the long measurement times needed (up to hours or even days).

To increase the measurement throughput of AFMs some smart measurement strategies have been developed. For instance, to reduce the calibration time of 2D gratings Dai et al. [4] proposed a calibration strategy where two crossed narrow bars are measured instead of a large size rectangular area; Ahmad A et al [5] developed an adaptive AFM scan speed control mechanism, where the lateral scanning speed of the AFM is adapted based on the scanner control error signal. However, the efficiency of these methods is still task relevant.

Generally speaking, there are two main approaches for enhancing the measurement speed of AFMs. One is to change the AFM measurement procedure from serial to parallel type [6]. This idea is usually realised by applying cantilever arrays, which are usually fabricated with integrated sensors and/or actuators using MEMS technology [7-8]. The shortcoming of this approach lies in the complicated fabrication of the sensors and the difficulties in its operation. The other approach is to enhance the measurement bandwidth of AFMs by realising, for instance, shorter response time of AFM sensing, faster scanner, faster signal detector and faster control techniques [9-12]. However, high speed AFMs developed till today are mainly applied in bioscience for, for instance, visualization of dynamic structural changes and dynamic processes of functioning biological molecules in physiological solutions [9]. These AFMs usually have limited scanning range (a few $\mu \mathrm{m}$ to tens of $\mu \mathrm{m}$ ). Imaging matching and stitching method is proposed to enlarge the measurement range of the AFM [13]. As an example, an interesting large area high-speed metrology AFM has been introduced [14] which is realised by combining a video rate AFM [15] with a large range mechanical stage (referred to as a nanopositioning and nanomeasuring machine, NMM [16]).

On the other hand, high speed AFMs developed till today focus mostly on the visualisation capability while their metrology capabilities are usually not concerned. A study was recently performed by Klapetek et al. [17] to map the measurement error of a high-speed AFM [15] using 1D gratings with pitch values of $100 \mathrm{~nm}$ and $300 \mathrm{~nm}$. They conclude that proper calibrations are needed for every scanning speed at which the AFM operates. In addition, strong image distortion, visible surface changes and debris caused by the high speed AFM measurements are shown.

A metrological high speed large range AFM (Met. HS-LRAFM) with a scanning speed up to 1 $\mathrm{mm} / \mathrm{s}$ has been recently developed at the Physikalisch-Technische Bundesanstalt, the national metrology institute of Germany. Both measurement performance concerning the speed and the accuracy are stressed in this development, as they are essential to realise fast and accurate nanometrology. In this paper, the design concept of the instrument is introduced briefly as they have been detailed in our previous publication [18]. The applications of the Met. HS-LRAFM in the field of surface and nanometrology are focused. 


\section{DESIGN CONCEPT}

A sample surface can be generally described as a 2D function as $S=f_{s}(x, y)$. AFM measures in the so-called scanning principle. When the sample is measured by an AFM in a fast scan axis of e.g. $x$, the surface can be depicted as collection of 1D functions:

$$
\left\{\left.z_{s}(x, y)\right|_{y=y_{i}}=z_{s}^{i}(x), \text { where } y_{i}=(i-1) \Delta y\right\}
$$

with $\Delta y$ being the space between scan lines.

If a AFM is scanning with a constant scanning speed $v$, the surface can also be written as a time signal as $z_{S}^{i}(x)=z_{S}^{i}(v t)$.

The general measurement principle AFM is shown in figure 1. For simplicity, we take the dynamic measurement mode as an example. A cantilever, which is oscillated near its resonance frequency, scans over the sample during measurements. The interaction force between the tip and sample leads to the change of the dynamic properties of the cantilever. After the bending and torsion of the cantilever is detected and demodulated, a real-time signal $z_{c}(t)$ representing the tip sample distance is obtained. The signal $z_{c}(t)$ is typically kept to be a constant value by a servo controller which moves the sample up and down using the scanner. Thus, the surface topography $z_{s}{ }^{i}(x)$ can be represented by the $3 \mathrm{D}$ motion of the scanner, measured by its position sensors as $x(t), z(t)$ at $y(t)=y_{i}$.

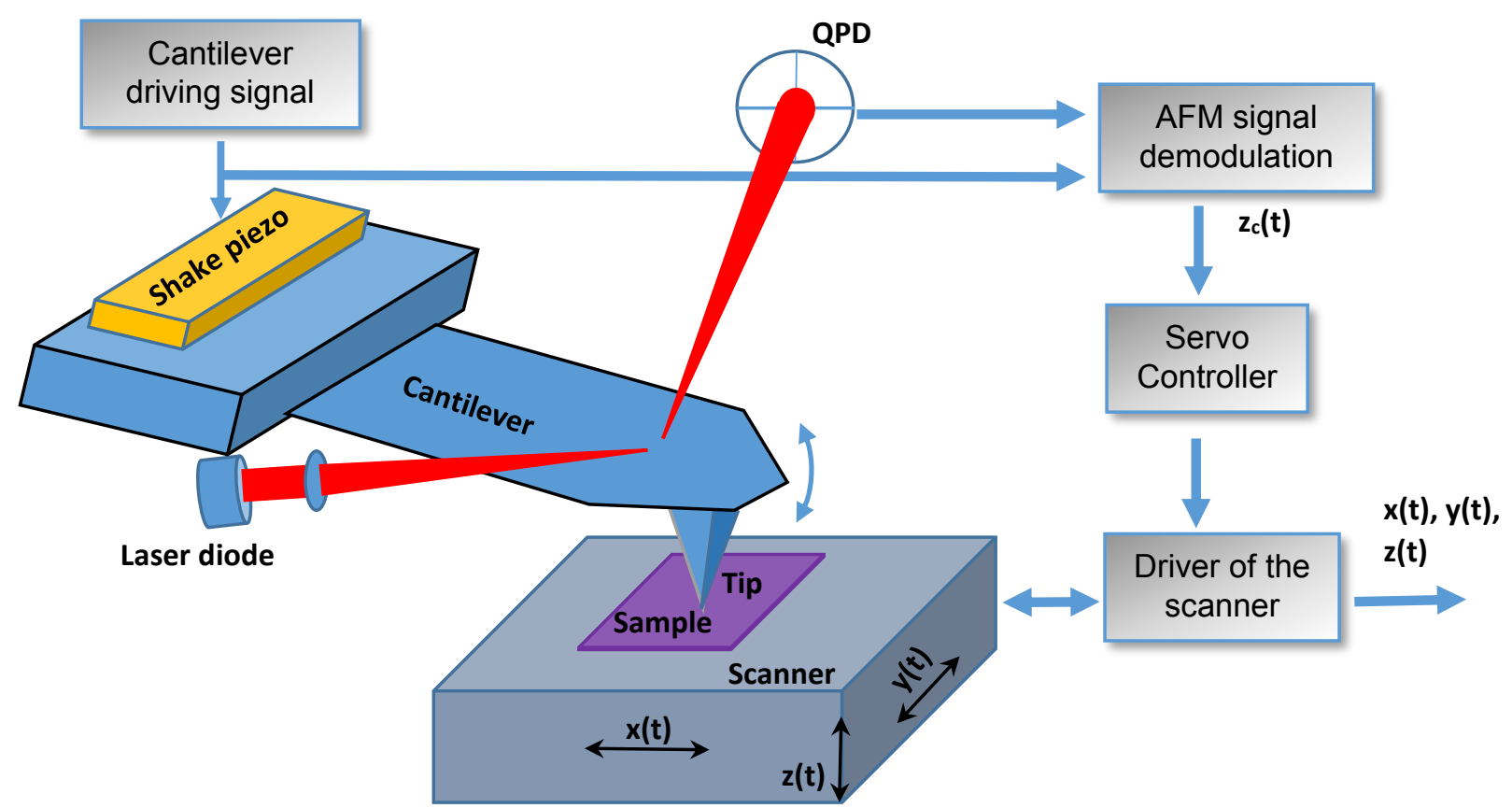

Figure 1. General measurement principle of an AFM working in the scanning sample principle.

For achieving a fast and accurate AFM measurement, there are three requirements: (i) the time response time of the AFM signal $z_{c}(t)$ should short enough so that the fast variation between the 
tip sample interaction can be correctly detected; (ii) the servo controller should be rapid enough to allow that the surface is well followed by the AFM tip; and (iii) the measured stage position $x(t), z(t)$ should be able to well represent the surface function, $z_{s}{ }^{i}(v t)$.

Let consider the signals $z(t)$ and $z_{s}{ }^{i}(v t)$ in the frequency domain by applying Fourier transformation:

$$
\begin{aligned}
\boldsymbol{Z}(\boldsymbol{\omega}) & =\mathscr{F}\{\mathrm{z}(\mathrm{t})\} \\
\text { and } \boldsymbol{G} s^{\prime}(\boldsymbol{\omega}) & =\mathscr{F}\left\{z_{S}^{i}(v t)\right\}=\frac{1}{|v|} \boldsymbol{G} \boldsymbol{s}\left(\frac{\boldsymbol{\omega}}{|v|}\right)
\end{aligned}
$$

where $\boldsymbol{G} \boldsymbol{s}(\boldsymbol{\omega})=F^{i}\left\{z_{S}{ }^{i}(x)\right\}$ is the spatial spectrum of the surface profile.

The equation (2) indicates that the signal spectrum of the surface to be measured is the spatial spectrum of the surface scaled by the scanning speed. Higher the scanning speed $v$, broader is the spectrum to be measured.

From the view point of the signal processing theory, to correctly represent the surface signal $z_{s}{ }^{i}(x)$ by the stage signal $x(t)$ and $z(t)$, the spectrum of the scanner $\boldsymbol{Z}(\boldsymbol{\omega})$ must cover the spectrum of the surface signal $\boldsymbol{G} s^{\prime}(\boldsymbol{\omega})$. This condition sets a basic limit on the measurement speed, as illustrated in figure 2. The spectrum of the stage is shown as a grey shadow area. It has a limited amplitude and bandwidth. The curves (i), (ii) and (iii) illustrate the spectrum $\boldsymbol{G} s^{\prime}(\boldsymbol{\omega})$ of a same surface when it is measured at different speeds. In the first two scenarios (i) and (ii), $\boldsymbol{G} s^{\prime}(\boldsymbol{\omega})$ is fully overlapped with $Z(\boldsymbol{\omega})$. It indicates that the surface signal can be fully presented by the stage motion, thus ensuring a correct measurement. However, in the scenario (iii), a significant part (indicated as $\varepsilon$ ) of the spectrum $\boldsymbol{G} s^{\prime}(\boldsymbol{\omega})$ is beyond the spectrum range of $\mathrm{Z}(\boldsymbol{\omega})$. As this part of signal can not be represented by the scanner motion, consequently a measurement error will occur. In addition, the scenario (iv) shows another measurement situation, where the height of the surface feature is larger than the motion range of the scanner, which also lead to measurement errors.

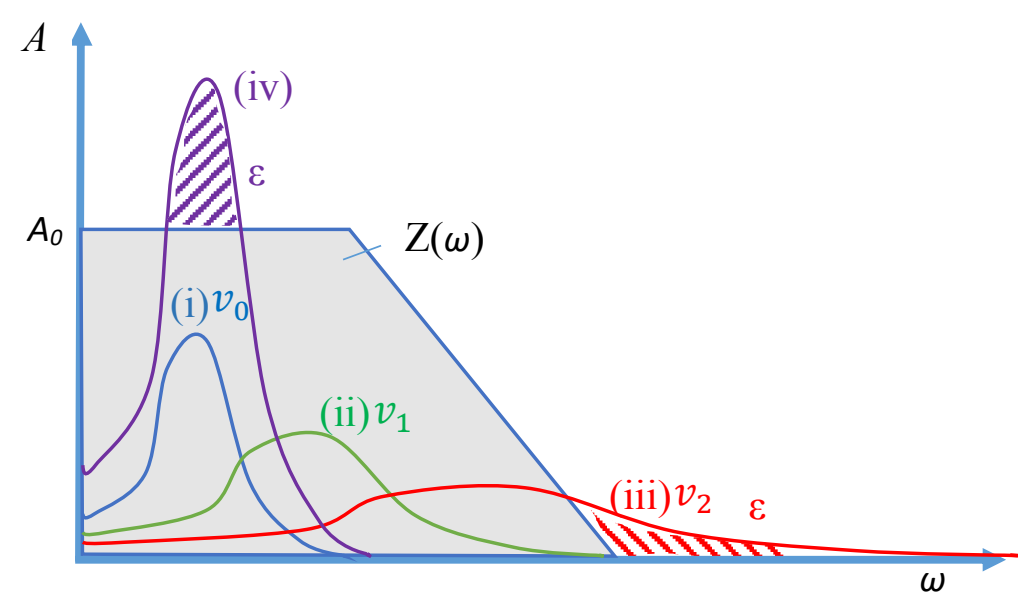

Figure 2. Limits of the measurement speed in the point of view in spectrum domain. 
It can be easily understood from figure 2 that an effective way to enhance the measurement speed is to enlarge the spectrum range of the scanner, $\boldsymbol{Z}(\boldsymbol{\omega})$, by increasing its dynamic performance. However, this usually needs more complicated design of the scanner.

PTB has recently developed a high speed metrological large range AFM (HS Met. LR-AFM) capable of both high speed and accurate measurements. Its measurement principle is shown in figure 3. Same as our previous Met. LR-AFM, the instrument is based on a large range mechanical stage referred to as a nanopositioning and nanomeasuring machine, NMM [16].

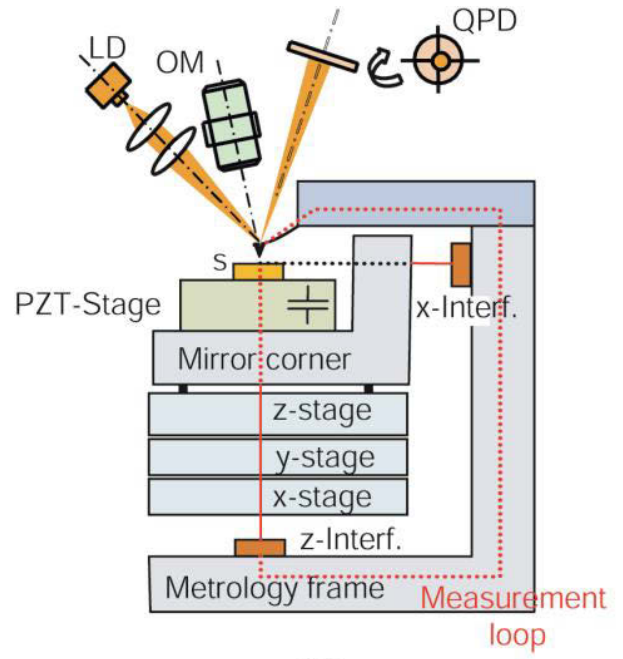

(a)

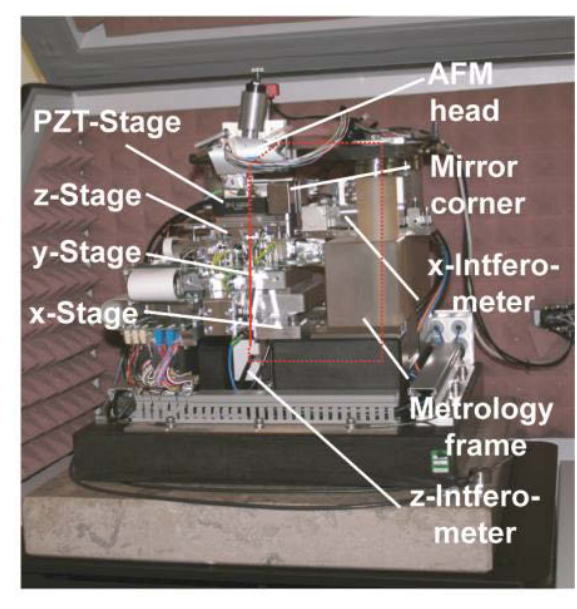

(b)

Figure 3. (a) Schematic diagram of the high-speed metrological large range AFM (High speed Met. LR-AFM); (b) photo of the instrument

Several important design concepts have been implemented to realise both, high measurement speed and high metrology performance:

- the contact AFM mode is applied instead of the intermittent and non-contact modes, which offers shorter AFM response time and larger AFM sensing range.

- during measurements, the sample is scanned in the xy-plane solely by the NMM (such a motion usually has a constant velocity, therefore, high dynamics of the xy-scanner is not needed.), however, a high dynamic $\mathrm{z}$ motion of the sample is realised by a combined piezo stage and the z-sage of the NMM controlled in parallel.

- the AFM output signal is combined with the position readouts of the piezo stage and the NMM to derive the surface topography. The combination of these readouts offers a large bandwidth of measurement signals, thus provides high speed measurement capability.

- two important means are taken to reduce the distortion in measured profiles, namely (a) the time delay of sensor signals are corrected; (b) the position sensors of the AFM and piezo stage are traceably calibrated to the z-interferometer of the NMM in situ.

Using such a measurement principle, The AFM output signal is combined with the positions of the piezo stage and the NMM to derive the surface topography $h(x)$ :

$$
z_{s}^{i}(v t)=S_{\text {zcap }}(t)+S_{A F M}(t)-S_{\text {zint } f}(t)
$$

Our design has a remarkable advantage that it well combines different bandwidth and amplitude of different stages/sensors. For instance, $Z_{\text {zint } f}(\boldsymbol{\omega})$ has a low bandwidth $<100 \mathrm{~Hz}$ (limited by 
the dynamic of the NMM) but a large amplitude up to several millimetres; $Z_{z c a p}(\boldsymbol{\omega})$ provide a middle bandwidth of approx. $300 \mathrm{~Hz}$ (limited by the dynamics of the piezo stage) and an amplitude range of several micrometres; while $Z_{A F M}(\boldsymbol{\omega})$ offers a high bandwidth of tens of $\mathrm{kHz}$ (or even higher) but a small amplitude range of up to hundreds of nanometres. The combination of the spectrums of these stages/sensors offers a much larger spectrum area, thus provides high speed measurement capability, as illustrated in figure 4.

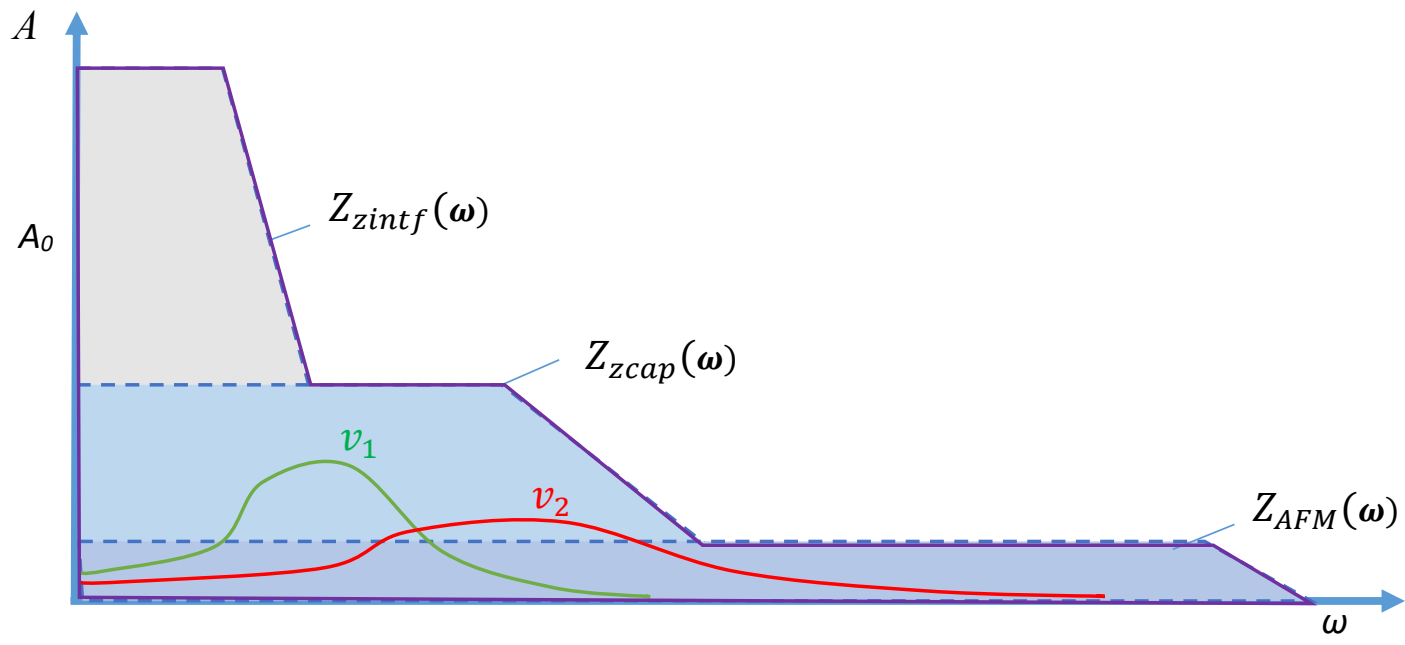

Figure 4. In the HS Met. LR-AFM, the measurable spectrum is the combination of the spectrums of the $N M M Z_{\text {zintf }}(\omega)$, the piezo stage $Z_{\text {zcap }}(\omega)$ and the $A F M$ sensor $Z_{A F M}(\omega)$. The combination of these spectrums offers a much larger spectrum area, thus provides the fast and accurate measurement capability.

For more detailed information about the instrumentation, please refer to our previous publication [18].

\section{MEASUREMENT PERFORMANCE}

To demonstrate the metrology performance of the high speed Met.LR-AFM, figure 5(a) shows a same surface profile measured at different speeds (from $10 \mu \mathrm{m} / \mathrm{s}$ up to $500 \mu \mathrm{m} / \mathrm{s}$ ). It can be seen the quality of measured profile is very similar despite the scanning speed has been increased by 50 times.

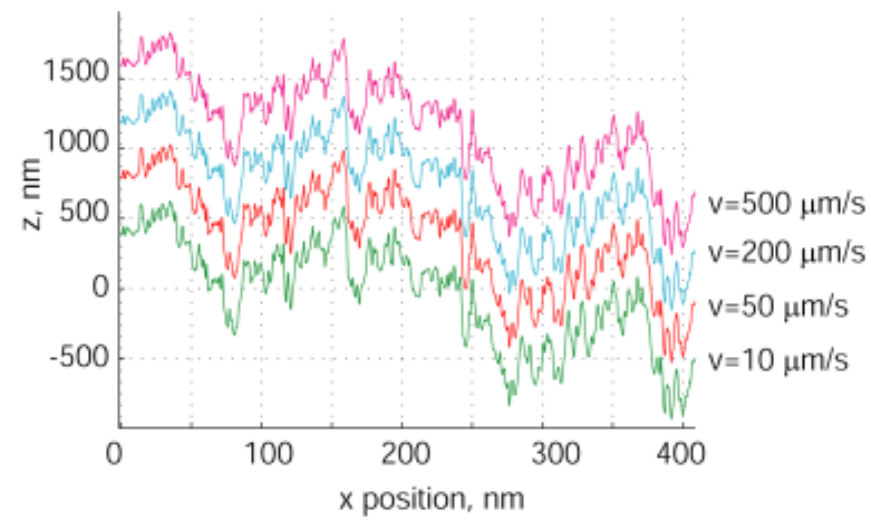

(a)

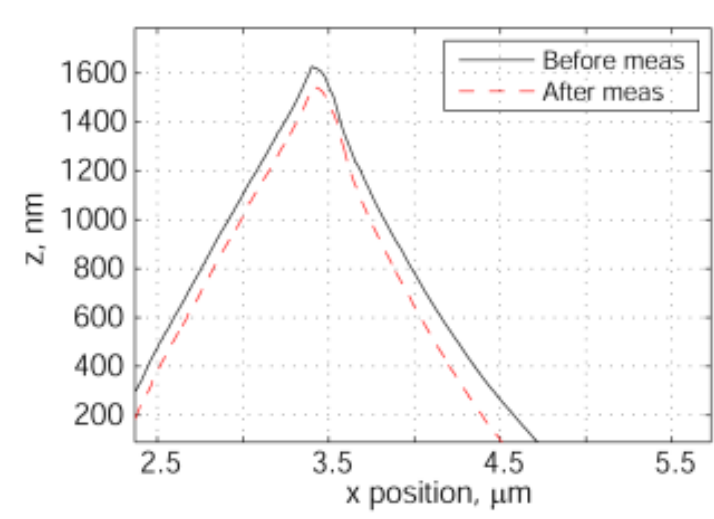

(b) 
Figure 5 Surface profiles taken on a PTB roughness standard (RN 505) by the high speed Met. $L R$-AFM, measured at the same location with different speeds from $10 \mu \mathrm{m} / \mathrm{s}$ up to $500 \mu \mathrm{m} / \mathrm{s}$; (b) Tip shape characterised before and after measurements. Profiles are intentionally shifted along the $y$-axis for clarity;

AFM tip wear is a critical issue, particularly for high speed AFM. In this study, AFM tip type ContDLC (Nanosensor), whose tip is coated with a diamond like carbon (DLC) layer, has been applied for AFM measurements. The tip geometry is characterised by a tip characteriser (a kind of sharp silicon triangular nanostructure) before and after the measurements, as shown in figure 5(b), indicating low tip wear.

\section{APPLICATION FOR REFERENCE SURFACE METROLOGY}

Today calibrations of areal surface measuring instruments are typically performed using a set of artefacts such as step height, grating, optical flats etc. for characterising the geometrical properties of the instruments (e.g. the scaling factors, linearities, squareness, flatness and noise [19-20]). Although being essential, these calibrations are still insufficient to fully characterise the measurement properties of the instruments, particularly the complicated probe sample interactions.

To overcome this challenging issue, a new idea of reference areal surface metrology is proposed in this paper. The reference metrology is to offer accurately and traceably calibrated reference 3D data maps of surfaces. By correlating the reference 3D data sets and the 3D data sets of tools under calibration, it is potential to comprehensively characterise the tools, for instance, its probe sample interaction and spectrum properties of tools.

Several optical surface measurement tools including confocal, white light interference and phase shift interference microscopes are investigated using the developed reference surface metrology. Due to the limited paper length, just a part of results is presented here.

Figure 6 shows the investigation result of a commercial confocal microscope referred as tool A with a 50x objective (tool's name is kept as anonymous for commercial reasons). Both measurements are taken on a same PTB roughness standard (SN505, nominal Ra of $184 \mathrm{~nm}$ ) with a same pixel density $(193.3 \mathrm{~nm} / \mathrm{pixel})$ and area size $(198 \mu \mathrm{m} \times 198 \mu \mathrm{m})$, thus allowing a direct comparison of two data sets in the same spectrum window. To account for the slight deviation of the measurement locations in two tools, the two data sets are aligned with each other using the cross-correlation algorithm. As the obtained areal surface topography images look quite similar, the comparison of a cross sectional profile of two data sets is illustrated in figure 6(a) with their discrepancies detailed in 6(b). It can be seen that the low frequency components of the surface in two data sets agree quite well, however, the optical results show significant artificial high frequency artefacts, especially in the valley and slope surface regions, which may be attributed to the optical probe sample interaction. In addition, a lateral shift of surface features (marked as A and B) is clearly visible at the left part of the image. It is probably due to the field distortion of the optical system, as the lateral scale of the microscope was properly calibrated prior to this investigation. Figure 6(c) shows the averaged power spectrum density (PSD) curves of the x-profiles of two data sets. Again, deviations for the components with frequencies $>0.21 / \mu \mathrm{m}$ are clearly visible. 


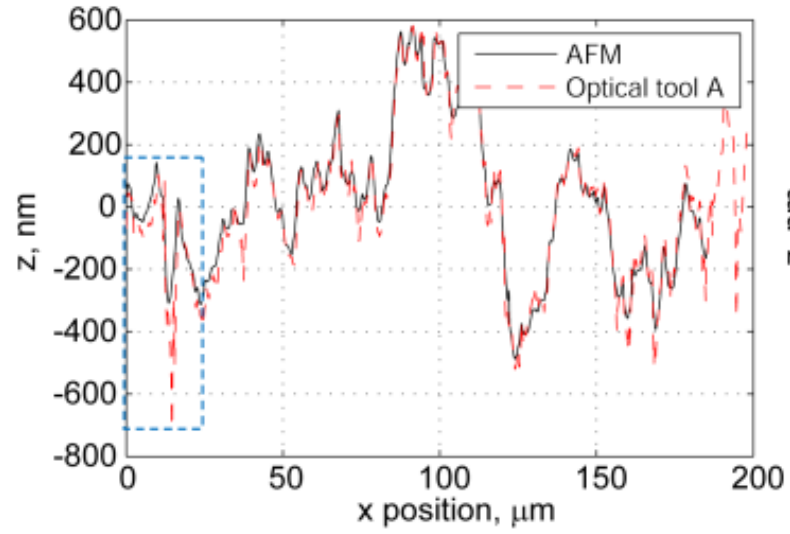

(a)

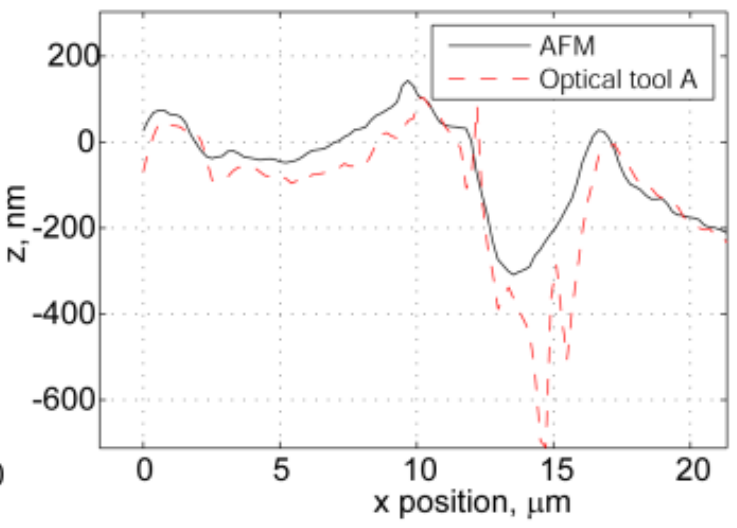

(b)

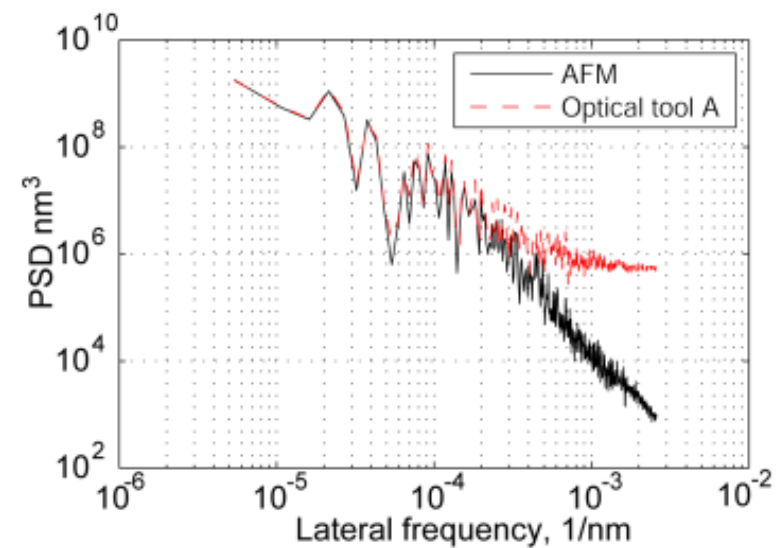

(c)

Figure 6. (a) comparison of a cross sectional profile of the measured reference data set and the data set measured by a commercial confocal microscope A; (b) detailed view of the discrepancies between profiles zoomed-in at the area marked in (a); (c) comparison of the averaged $1 D$ power spectrum density (PSD) curve of two data sets.

Similar investigations have been performed on another commercial confocal microscope referred as tool B with a 50x objective, pixel density of $331 \mathrm{~nm} /$ pixel and field size of $254 \mu \mathrm{m}$ x $190 \mu \mathrm{m}$. The measurement and data processing are the same as that of the previous study. Again, a cross sectional profile of two data sets is compared in figure 7(a) and the averaged PSD curves of x-profiles of two data sets compared in figure 7(b), showing surprisingly good agreement.

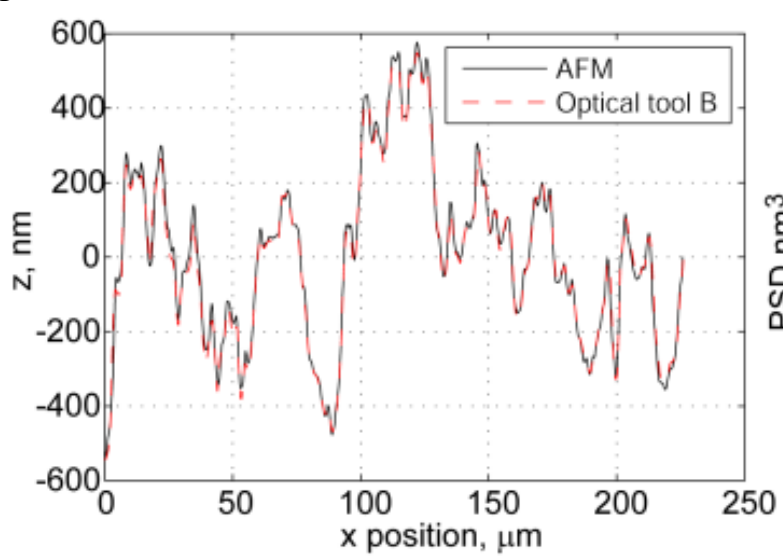

(a)

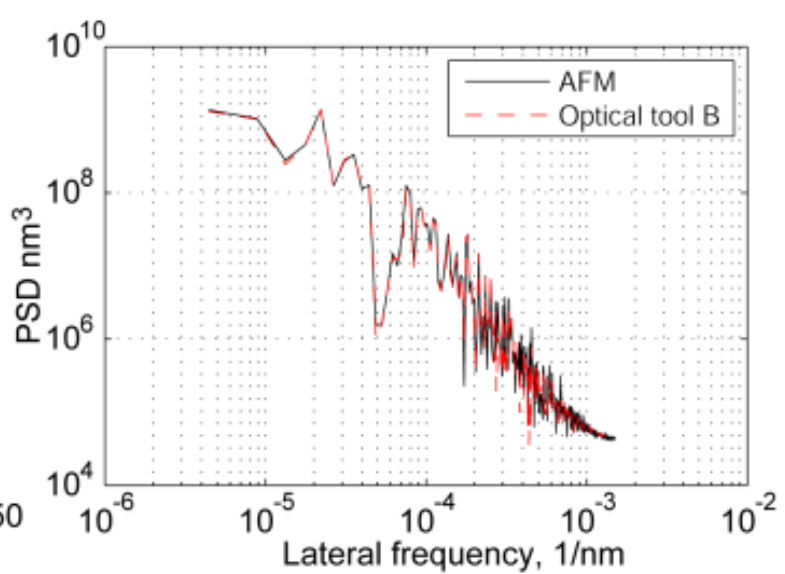

(b) 
Figure 7. (a) comparison of a cross sectional profile of the measured reference data set and the data set measured by a commercial confocal microscope B; (c) comparison of the averaged 1D power spectrum density (PSD) curve of two data sets.

\section{APPLICATION FOR THE CALIBRATION OF 3D NANO STANDARDS}

The second application example presented here is the calibration of a kind 3D nano standard developed by the company $\mathrm{m}^{2} \mathrm{c}$ [21]. The standard has a shape of a cascade slope-step pyramid, on which a number of landmarks with sub-micrometre size, the so-called 'nanomarkers' are fabricated. Prior to its usage, the 3D coordinates of nanomarkers are accurately and traceably calibrated using the Met. LR-AFM at PTB. The standard is then measured by a user instrument and its obtained 3D coordinates of nanomarkers are compared to the calibrated reference data, thus the 3D geometrical errors of the user instrument can be calibrated. In contrast to classical calibration procedures that require separate lateral and vertical reference standards such as gratings and step height structures, this calibration method needs only one 3D nano standard for calibrating the full 3D geometric errors, including parameters of three scale factors (for $\mathrm{x}$, $\mathrm{y}$ and $\mathrm{z}$ respectively) and three coupling factors ( $\mathrm{x}$ to $\mathrm{y}, \mathrm{y}$ to $\mathrm{z}$ and $\mathrm{x}$ to $\mathrm{z}$, respectively). The method has been successfully established and applied for calibrating versatile microscopic techniques, such as scanning probe microscopes, scanning electron microscopes and confocal laser scanning microscopes.

To ensure a fine measurement resolution of nanomarkers, the 3D nano standard was measured with a typical scan speed of $10 \mu \mathrm{m} / \mathrm{s}$, which takes more than two hours to obtain one image. With the developed HS Met. LR-AFM, we have so far performed at a scan speed of $100 \mu \mathrm{m} / \mathrm{s}$, i.e. ten times faster, thus dramatically reduce the calibration costs.

To demonstrate the calibration performance, figure 7 depicts an AFM image of a 3D nano standard (MMC160-ID601) measured at the scan speed of $100 \mu \mathrm{m} / \mathrm{s}$ shown as a 3D view. The size of the image is $180 \mu \mathrm{m} \times 180 \mu \mathrm{m} \times 5.4 \mu \mathrm{m}$ (x, y and z) and 1024 pixels $\times 1024$ pixels, shown with a scale ratio of $1: 1: 1$. It can be seen in the image that both the pyramid and the nanomarkers are well resolved. 


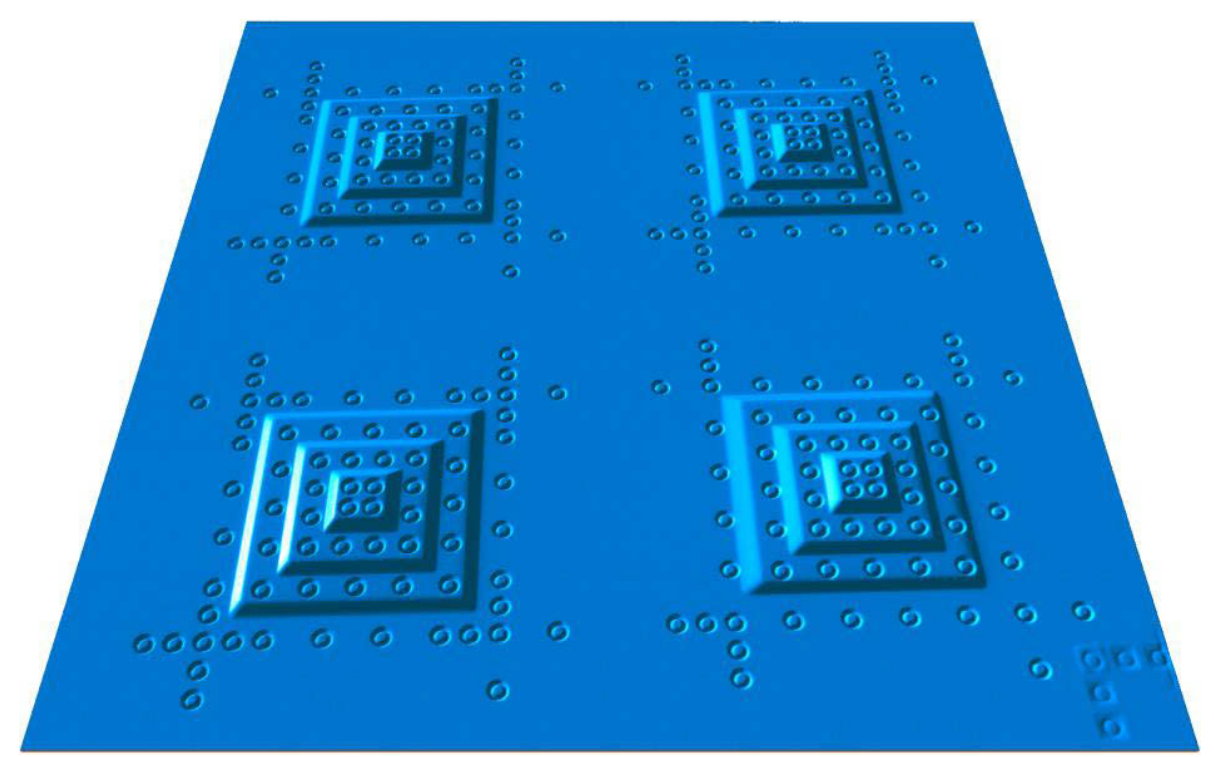

Figure 7. A typical AFM image measured on a 3D nano standard (MMC160-ID601) at the scan speed of $100 \mu \mathrm{m} / \mathrm{s}$ shown as a $3 D$ view. The size of the image is $180 \mu \mathrm{m} \times 180 \mu \mathrm{m} \times 5.4$ $\mu m$ ( $x, y$ and $z$ ), sampled with 1024 pixels $x 1024$ pixels, and shown with a scale ratio of 1:1:1.

To investigate the measurement repeatability, the sample is measured when it is mounted in two different orientations, where the sample is rotated by 90 degrees anticlockwisely in the $2^{\text {nd }}$ orientation with respect to the $1^{\text {st }}$ measurement. The obtained $3 \mathrm{D}$ coordinates of the $2^{\text {nd }}$ measurement of nanomarkers are then $3 \mathrm{D}$ rotated and matched to the $1^{\text {st }}$ set. The residual matching error in the xy plane is shown in figure 8(a), and that along the z-direction is shown in figure $8(\mathrm{~b})$, respectively. The scale of the error bar is magnified by a factor of 100 with respect to the scale of the image for clarity.

The mean matching errors are $23.1 \mathrm{~nm}, 17.0 \mathrm{~nm}$ and $4.3 \mathrm{~nm}$ along the $\mathrm{x}, \mathrm{y}$ and $\mathrm{z}$ axis, respectively. It is a satisfactory result, and is similar to our previous results measured at a slow scan speed of $10 \mu \mathrm{m} / \mathrm{s}$. Using the high speed AFM mode, we could dramatically reduce the calibration time, thus the costs. 


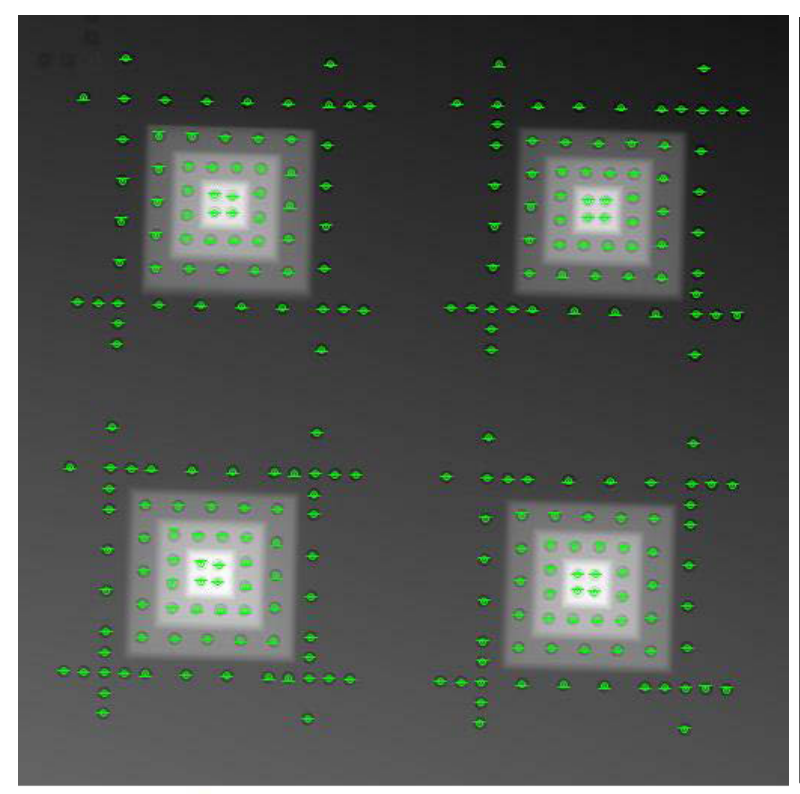

$\longmapsto 10 \mu \mathrm{m} \quad(0.1 \mu \mathrm{m})$

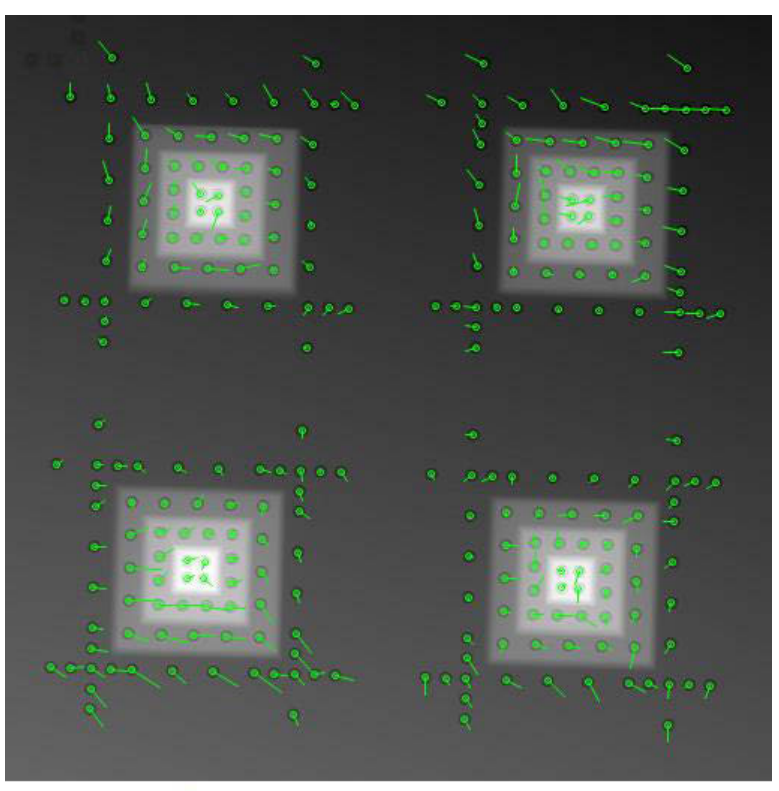

$\longmapsto 10 \mu \mathrm{m} \quad(0.1 \mu \mathrm{m})$

(a)

(b)

Figure 8. the matching error of two $3 D$ coordinate sets of nanomarkers which were measured with sample mounted in two different orientations. The residual matching error vectors in the $x y$ plane is shown in figure $8(a)$, and that along the z-direction is shown in figure 8(b), respectively. The scale of the error bar is magnified by a factor of 100 with respect to the scale of the image for clarity.

\section{CONCLUSION}

Metrological large-range atomic force microscope (Met. LR-AFM) with a capable measurement volume up to $25 \mathrm{~mm}$ x $25 \mathrm{~mm}$ x $5 \mathrm{~mm}(\mathrm{x}, \mathrm{y}, \mathrm{z})$ has been developed in the last decade based on high precision positioning stage, e. g. the well-known nanopositioning and measuring machine (NMM). However, till now the low measurement speed remains as a major shortcoming of the Met. LR-AFM. It leads not only to a low measurement throughput, but also to a significant drift over the long measurement times needed (up to hours or even days). In this paper, we present a method for improving the measurement speed up to $1 \mathrm{~mm} / \mathrm{s}$, and versatile applications for accurate surface and nanometrology.

Several design ideas are implemented to realise fast and accurate measurement. Firstly, a piezo stage and a NMM is combined to move the sample in parallel along the $\mathrm{z}$-axis, thus providing both a high dynamic positioning capability and a large motion range. Secondly, a contact mode AFM which offers both, a short response time and a large sensing range is applied for measuring the surfaces of interest. Sensor signals from the AFM, the piezo stage and the NMM are combined to derive the surface topography, thus offering high bandwidth for high speed measurements. Thirdly, to reduce the distortion in the measured profiles, the time delay of sensors is corrected as well as the displacements of the AFM and the piezo stage are traceably 
calibrated referring to the z-interferometer of the NMM in situ. Experimental results demonstrate the high image quality obtainable at a scan speed of $500 \mu \mathrm{m} / \mathrm{s}$. The measurement repeatability at different scan speeds ranging from $10 \mu \mathrm{m} / \mathrm{s}$ to $1000 \mu \mathrm{m} / \mathrm{s}$ reaches $\pm 0.7 \mathrm{~nm}$ for step height measurements and $\pm 5 \times 10$ - 6 for grating pitch measurements, respectively. The study has improved the measurement speed of the Met. LR-AFM by a factor of more than 20 times.

Two application examples of the developed high speed LR-AFM are demonstrated in the paper. The first example is the realisation of reference areal surface metrology. Today calibrations of areal surface topography measuring instruments are typically performed using a set of artefacts such as step height, grating, optical flats etc. for characterising the geometrical properties of the instruments (e.g. the scaling factors, linearities, squareness, flatness and noise). Although being essential, these calibrations are still insufficient to fully characterise the transfer function of the instruments, particularly the complicated probe sample interactions. To overcome this challenging issue, a new idea of reference areal surface metrology is proposed in this paper. The reference metrology is to offer accurately and traceably calibrated reference 3D data maps of surfaces. By correlating the reference $3 \mathrm{D}$ data sets and the 3D data sets of tools under calibration, it is potential to comprehensively characterise the tools, for instance, its probe sample interaction and spectrum properties of tools. Several optical surface measurement tools including confocal, white light interference and phase shift interference microscopes are investigated using the developed reference areal surface metrology, indicating promising results.

The second example is the calibration of a kind 3D nano standards which have spatially distributed landmarks, i.e. special unique features defined by $3 \mathrm{D}$-coordinates. Compared to conventional step height and lateral standards, the 3D nano standards are applicable for calibrating 3D geometric errors of microscopes with only one measurement, which is accomplished by comparing the spatial coordinates of landmarks measured by customer instruments to the its reference coordinates calibrated by the metrological LR-AFM. Because of the low scanning speed (typically of $20 \mu \mathrm{m} / \mathrm{s}$ ) in our previous instrument, it usually took approximately 3 to 4 hours for one measurement and a weekend for a whole calibration. The application of the high-speed LR-AFM thus significantly improved the calibration efficiency, thus reducing the calibration costs. A number of experimental investigations confirmed that calibration accuracy is maintained at higher measurement speeds.

\section{ACKNOWLEDGEMENT}

The work has received funding from the EMPIR programme co-financed by the Participating States and from the European Union's Horizon 2020 research and innovation programme, project 15SIB09 3Dnano.

\section{REFERENCES}

[1] G. Binning, R. G. Rohrer, C. Gerber, and E. G. Weibel 1982 Surface Studies by Scanning Tunneling Microscopy, Phys. Rev. Lett. 49, 57. 
[2] G. Binning, C. F. Quate, and C. Gerber 1986 Atomic Force Microscope, Phys. Rev. Lett. 56,930 .

[3] Gaoliang Dai,Helmut Wolff, Frank Pohlenz et al. 2009 A metrological large range atomic force microscope with improved performance, Review of Scientific Instruments 80, 043702

[4] Gaoliang Dai, Frank Pohlenz, Thorsten Dziomba et al. 2007 Accurate and traceable calibration of two-dimensional gratings, Meas. Sci. Technol. 18: 415-421

[5] Ahmad A, Schuh A, Rangelow IW, 2014 Adaptive AFM scan speed control for high aspect ratio fast structure tracking, Rev Sci Instrum 85(10): 103706

[6] Minne S C, Yaralioglu G, Manalis S R, et al 1998 Automated parallel high-speed atomic force microscopy, Appl. Phys. Lett., 72(18), 2340-2

[7] Ahn Y, Ono T, Esashi M 2008 Micromachined Si cantilever arrays for parallel AFM operation, Journal of Mechanical Science and Technology 22(2), 308-311

[8] Sulzbacha T, Engla W, Maiera R et al 2010 Cantilever arrays with integrated actuation and sensing for parallel SPM, Procedia Engineering 5, 621-624

[9] Ando T 2012 High-speed atomic force microscopy coming of age, Nanotechnology 23 062001

[10] Kodera N, Yamashita H and Ando T 2005 Active damping of the scanner for high-speed atomic force microscopy, Rev. Sci. Instrum. 76, 053708

[11] Yong Y K, Moheimani S O R, Kenton B J and Leang K K 2012 Invited review article: High-speed flexure-guided nanopositioning: mechanical design and control issues, Review of Scientific Instruments, 83 (12), 121101

[12] Schitter G and Rost M J 2008 Scanning probe microscopy at video-rate, Materials Today, Volume 11, Supplement, 2008, Pages 40-48

[13] Hobbs J K, Vasilev C, Humphris A D L 2006 VideoAFM-a new tool for high speed surface analysis, Analyst, 131, 251-256

[14] Klapetek P, Valtr M, Picco L et al 2015 Large area high-speed metrology SPM system, Nanotechnology 26, 065501

[15] Picco L M, Dunton P G, Ulcinas A et al 2008 High-speed AFM of human chromosomes in liquid, Nanotechnology 19, 384018

[16] Manske E, Hausotte T, Mastylo R, et al 2007 New applications of the nanopositioning and nanomeasuring machine by using advanced tactile and non-tactile probes Meas. Sci. Technol. 18, 520

[17] Klapetek P, Picco L, Payton O et al. 2013 Error mapping of high-speed AFM systems, Meas. Sci. Technol. 24025006

[18] Gaoliang Dai, Fan Zhu and Jens Fluegge 2015 High-speed metrological large range AFM, Meas. Sci. Technol. 26: 095402

[19] Giusca C L, Leach R K, Helary F et al. 2012 Calibration of the scales of areal surface topography-measuring instruments: part 1. Measurement noise and residual flatness, Meas. Sci. Technol. 23035008

[20] Giusca C L, Leach R K, Helary F 2012 Calibration of the scales of areal surface topography measuring instruments: part 2 . Amplification, linearity and squareness, Meas. Sci. Technol. 23065005

[21] Martin Ritter, Thorsten Dziomba, Axel Kranzmannand Ludger Koenders 2007 A landmark-based 3D calibration strategy for SPM, Meas. Sci. Technol.18 404-414

\section{CONTACTS}

